\title{
Importancia de la vegetación ribereña de Nothofagus dombeyi (Mirb.) Oerst. en el régimen térmico de sistemas fluviales andinos del sur de Chile
}

\section{The importance of Nothofagus dombeyi (Mirb.) Oerst. riparian vegetation in the thermal regime of Andean streams of Southern Chile}

\author{
Pablo Pedreros $^{1 *}$, Meyer Guevara-Mora ${ }^{1}$, Roberto Urrutia ${ }^{1} \&$ Alejandra Stehr $^{2}$ \\ ${ }^{1}$ Departamento de Sistemas Acuáticos, Facultad de Ciencias Ambientales, Centro EULA, Universidad de Concepción, Casilla \\ 160-C, Concepción, Chile. \\ ${ }^{2}$ Departamento de Ingeniería Ambiental, Facultad de Ciencias Ambientales, Centro EULA, Universidad de Concepción, Casilla \\ 160-C, Concepción, Chile. \\ *papedrer@udec.cl
}

\begin{abstract}
RESUMEN
A nivel global la temperatura de los ríos sigue siendo tema de investigación en ecología fluvial, pero el conocimiento de su variabilidad espacio-temporal en diversos sistemas fluviales aún es limitada y en algunos casos desconocida. Este estudio tuvo por objetivo comparar el régimen térmico natural de dos ecosistemas fluviales andinos con diferente vegetación ribereña (estepa y bosque de Nothofagus dombeyi). La temperatura en ambos sitios fue registrada con una resolución de 15 min desde enero a julio de 2011. La temperatura media, máxima y rango diario del agua mostraron diferencias significativas, con valores más bajos en el río con presencia de $N$. dombeyi. En relación al ciclo diario, se observó un leve retraso en la temperatura mínima y adelanto en la máxima para el río con bosque nativo. La realización de monitoreos continuos mediante sensores de temperatura permitió determinar contrastes en el régimen térmico y sugiere que la sombra generada por el dosel reduce la radiación solar que recibe el río y la temperatura máxima $\left( \pm 4{ }^{\circ} \mathrm{C}\right)$ en ríos andinos. Asimismo, la conservación de la cobertura boscosa de $N$. dombeyi en la zona andina podría ser importante para moderar y mitigar la ocurrencia de temperaturas más altas a consecuencia de los escenarios de calentamiento global proyectados para la zona.
\end{abstract}

Palabras clave: Bosque nativo, estepa, temperatura fluvial.

\begin{abstract}
Globally, the river temperatures is a research subject, but the spatio-temporal variability of various river systems is still limited and unknown in some cases. This study aimed to compare the natural thermal regime of Andean river ecosystems with different riparian vegetation (steppe and Nothofagus dombeyi forest). The temperature of the sites was registered with a 15 min time resolution from January to July 2011. The average, maximum and daily temperature range of water showed significant differences, with lower values in the presence of $N$. dombeyi. Regarding the daily cycle, a slight delay was observed in the minimum temperature and an advance in the maximum temperature for the native forest site. Therefore, performing continuous monitoring using temperature sensors made it possible to determine contrasts in the thermal regime suggesting that the shade generated by the canopy reduces the solar radiation and the maximum temperature $\left( \pm 4{ }^{\circ} \mathrm{C}\right)$ in Andean rivers. Furthermore, the conservation of forest cover of $N$. dombeyi in the Andean region might be important to moderate and mitigate the occurrence of higher temperatures due to global warming scenarios projected for the area.
\end{abstract}

KEYWORDS: Native forest, steppe, river temperature.

\section{INTRODUCCIÓN}

El régimen térmico característico de un paisaje fluvial responde a una combinación de entradas de energía desde diversas fuentes, incluidos factores externos y estructuras internas que se relacionan y determinan a corto o largo plazo las tendencias térmicas (Poole \& Berman 2001, Caissie 2006, Webb et al. 2008). En situaciones naturales, las condiciones meteorológicas han sido reconocidas como el principal modelador de la temperatura, siendo la radiación solar de 
onda corta el factor dominante (Sinokrot \& Stefan 1993, Webb \& Zhang 1997, Johnson 2003). No obstante, también existen factores topográficos y morfológicos del cauce que modelan la variabilidad térmica (Poole \& Berman 2001). Al respecto, estudios desarrollados en ríos de cabecera del Hemisferio Norte destacan la importancia de la vegetación ribereña sobre la dinámica térmica (Johnson \& Jones 2000, MacDonald et al. 2003, Danehy et al. 2005, Moore et al. 2005b, Malcolm et al. 2008). Su presencia afecta a la transmisión de la radiación incidente al medio acuático, así como a otros factores meteorológicos (e.g. viento, humedad y temperatura del aire) que contribuyen al intercambio de calor neto (Moore et al. 2005a, Hannah et al. 2008). De acuerdo a Poole \& Berman (2001), un denso dosel arbóreo reduce en un $90 \%$ la radiación solar incidente. Similarmente, Malcolm et al. (2004), Rutherford et al. (2004) y Webb \& Crisp (2006) demostraron que la tasa de calentamiento y las fluctuaciones térmicas diarias responden a la secuencia de sombra generada por el dosel ripario.

A pesar de la importancia que tiene la vegetación ribereña en el control del régimen térmico fluvial, en muchos ríos se ha modificado la vegetación aledaña al cauce, particularmente a consecuencia del desarrollo de la actividad agrícola y forestal (Webb et al. 2008). Dicha alteración ha provocado un aumento en la entrada de calor, lo que se traduce en una modificación de los patrones térmicos del agua alterando el funcionamiento fluvial (Vannote \& Sweeney 1980, Berner \& Berner 1996, Acuña et al. 2008, Quinn \& Wrightstow 2008). En Chile, a pesar del marcado gradiente térmico latitudinal-altitudinal existente, los estudios de régimen térmico en ecosistemas fluviales son limitados y orientados principalmente a tramos medios y bajos de ríos fuertemente influenciados por la actividad humana (Link et al. 2009, 2012, Monsalve et al. 2012). Recientemente Pedreros et al. (2013) describieron que la heterogeneidad térmica de los ríos andinos está regulada principalmente por sus características geomorfológicas, destacando la importancia de las altas montañas en la generación de sombra. Si bien la degradación del paisaje ecológico ha sido notoria desde tiempos de la conquista y colonización (Cisternas et al. 1999, Torrejón \& Cisternas 2002), durante las últimas décadas ha experimentado un mayor impacto, no sólo en el valle central sino también hacia las zonas andinas. Así Aguayo et al. (2009) muestran que la pérdida de bosque nativo de Nothofagus spp. en los cordones montañosos andinos se debe, en gran medida, al desarrollo forestal, habilitación de terrenos agropecuarios y crecimiento urbano e industrial. Tal modificación en la vegetación ribereña nativa podría alterar la temperatura de los ríos andinos sin que sus efectos sean previamente evaluados, provocando una modificación importante en el funcionamiento de estos sistemas acuáticos, dado que dicha variable controla procesos claves de la ecología fluvial (Allan \& Castillo 2007).
Por lo tanto, considerando que la sombra generada por las altas montañas es un factor relevante en regular la temperatura de ríos alto andinos del centro sur de Chile, es probable que la heterogeneidad térmica también sea regulada por la secuencia de sombra generada por la presencia de bosque de Nothofagus dombeyi en dichos sistemas fluviales. Así el objetivo principal del presente trabajo fue comparar y describir el régimen térmico (rango térmico diario, temperatura media, mínima y máxima diaria) de dos ecosistemas fluviales con vegetación ribereña contrastante en la cuenca del río Biobío, mediante registros térmicos constantes. Además se evaluó la relación entre la temperatura del agua y la temperatura de aire con el propósito de analizar el efecto de esta última variable en cada sitio de estudio.

\section{MATERIALES Y MÉTODOS}

El área de estudio corresponde al sector andino de la cuenca del río Biobío (Fig. 1a y b) y considera los ríos:

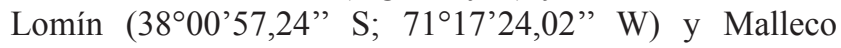
(38 $12^{\prime} 50,17^{\prime}$ ' S; 71 $\left.{ }^{\circ} 48^{\prime} 21,68^{\prime \prime} \mathrm{W}\right)$. Ambos sistemas fluviales presentan características de climas mediterráneos (Gasith \& Resh 1999) y se encuentran inmersos en la zona fluvio-glacio-volcánica (Mardones et al. 1992). La cuenca del río Lomín está constituida mayoritariamente por un conglomerado de rocas ígneas con intrusiones sedimentarias, al contrario de la cuenca del río Malleco donde dominan las rocas sedimentarias e ígneas con intrusiones sedimentarias. En ambos casos, el régimen hidrológico puede ser descrito como pluvio-nival con crecidas importantes durante invierno y primavera. Mientras que el sustrato del cauce está compuesto mayoritariamente por un conjunto de cantos rodados, piedras y gravas.

Ambos sistemas presentan un tipo de vegetación contrastante (Fig. 1 c). El río Lomín drena principalmente sectores dominados por estepa alto andina subhúmeda, no obstante existe un continuo de renoval ribereño nativo compuesto mayoritariamente por coigüe (Nothofagus dombeyi (Mirb.) Oerst) y ñirre (N. antarctica (G. Forst.) Oerst) en su tramo final. Contrariamente, el río Malleco drena áreas dominadas por una vegetación ribereña nativa constituida principalmente por la especie perenne Nothofagus dombeyi y, en menor proporción, por mirtáceas (e.g. Myrceugenia ovata (Hook. et Arn.) Berg.) y poáceas (e.g. Chusquea quila (Kunth)). Además, cada sitio de estudio fue caracterizado incluyendo datos geográficos y características físicas del río (ver Tabla I). El área de drenaje y orden del río de cada sitio de muestreo fue obtenida de las bases de datos cartográficas del IGM (Instituto Geográfico Militar; 1:50.000). Adicionalmente también se obtuvo la altitud mínima, máxima y media; y pendiente media de cada subcuenca. 
RECOLECCIÓN DE DATOS

Los datos de campo fueron obtenidos entre enero y julio de 2011. La temperatura del aire y del agua para ambos sitios se midió continuamente cada $15 \mathrm{~min}$. Para ello se utilizaron sensores de temperatura marca HOBO modelo UA-001-08 $\left(-20\right.$ a $\left.70{ }^{\circ} \mathrm{C}\right)$, con una precisión de $\pm 0,5^{\circ} \mathrm{C}$. Para estimar la temperatura del agua, en cada sitio se instaló un sensor en la sección media del río, dado que no existieron diferencias significativas en la sección transversal del cauce $(P<0,05)$. Previo a su instalación los sensores fueron programados y calibrados $24 \mathrm{~h}$ antes, de acuerdo a la metodología de Pedreros et al. (2013). Una vez calibrados estos termistores fueron introducidos en tubos de PVC (diámetro $=35 \mathrm{~mm}$; longitud $=15 \mathrm{~cm}$ ) a fin de evitar que recibieran radiación directa que alterara la temperatura del sensor. El ancho máximo y la profundidad media de cada río se estimó in situ utilizando una cinta métrica y vara graduada (Tabla I).

\section{ANÁLISIS ESTADÍSTICO}

A partir de los registros de temperatura del aire y del agua se calcularon los siguientes parámetros térmicos: (i) temperatura media diaria, (ii) temperatura mínima diaria, (iii) temperatura máxima diaria y (iv) rango medio diario. Este último fue calculado como la diferencia entre la temperatura máxima y mínima diarias. El software utilizado para descargar los registros de temperatura fue HOBOware Pro versión 2.7. Para establecer la relación entre la temperatura del aire y del agua de ambos sitios se calculó el coeficiente de correlación de Pearson $(P<0,05)$, utilizando los datos con intervalos de $15 \mathrm{~min}$. Con el objetivo de proporcionar una visión más detallada de las diferencias de temperatura entre los sitios con bosque y estepa se realizó una comparación a escala temporal diaria empleando el set de datos de alta resolución (15 min) en dos períodos de $24 \mathrm{~h}$; uno para el verano (día más cálido) y otro para otoño (día a)

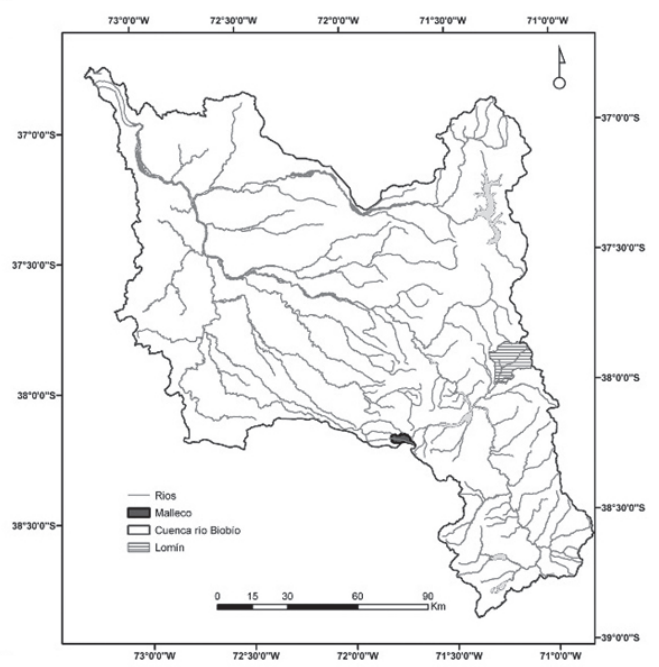

c)

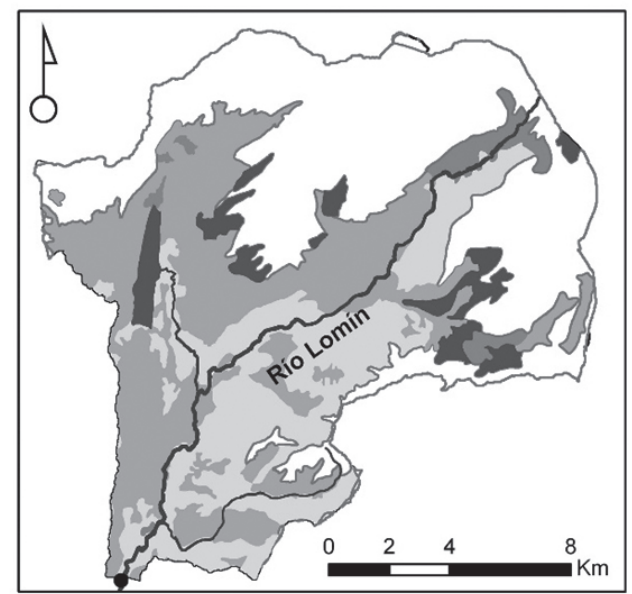

b)

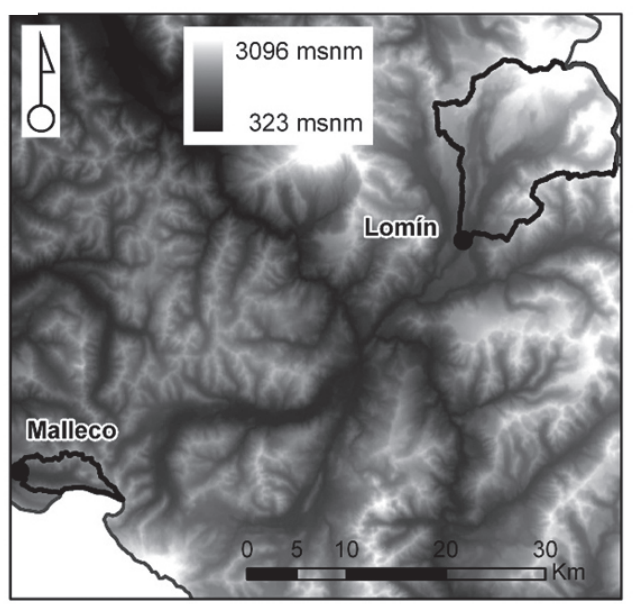

d)

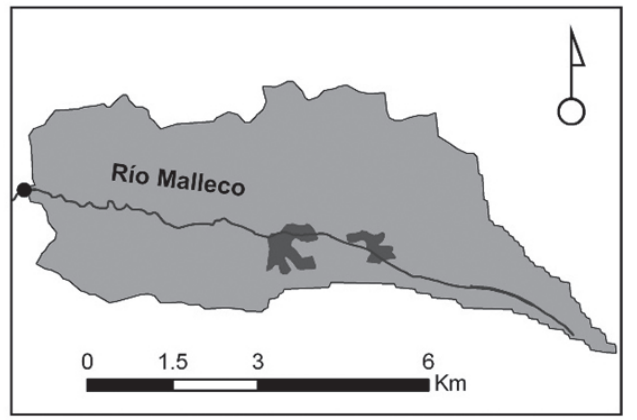

Tipos de vegetación

Estepa Andina Bosque Nativo Matorrales Glaciares/Nieve Otros

Figura 1. a) Localización del área de estudio. b) Modelo de elevación digital. c) y d) Distribución generalizada del tipo de vegetación de las cuencas de los ríos Lomín y Malleco, respectivamente. Los círculos negros corresponden a los sitios de estudio.

Figure 1. a) Location of the study area. b) Digital elevation model. c) y d) Generalized distribution of vegetation for Lomín and Malleco watersheds. Black circle correspond to sampling sites. 
más frío), basados en la temperatura máxima y mínima del aire registrada el 23 enero y 25 junio, respectivamente. La prueba no paramétrica U-Mann Whitney $(P<0,05)$ fue utilizada para establecer diferencias significativas en el ciclo diario de temperatura (aire y agua) entre los sitios de estudio. Todos los análisis fueron realizados utilizando software $\mathrm{R}$ (Fox 2005). Finalmente, para establecer la influencia de la energía térmica disponible para organismos acuáticos se calcularon los grados-día acumulados y mensuales desde febrero a julio. De acuerdo a Arscott et al. (2001), este parámetro corresponde a la sumatoria de las temperaturas medias diarias sobre $0^{\circ} \mathrm{C}$ y ha mostrado importancia en el desarrollo metabólico de diversos grupos taxonómicos (Olden \& Naiman 2010).

\section{RESULTADOS}

La temperatura media diaria del aire registró una tendencia decreciente con patrones térmicos similares no significativos $(\mathrm{U}=14.128 ; P=0,28)$ entre el sitio con bosque nativo $\left(8,15^{\circ} \mathrm{C}\right)$ y estepa $\left(8,73^{\circ} \mathrm{C}\right)$ (Tabla II). En tanto, las temperaturas máximas y mínimas diarias registraron valores de $21,52{ }^{\circ} \mathrm{C}$ y $-3,39{ }^{\circ} \mathrm{C}$ para el río Malleco (bosque) y de $22,33{ }^{\circ} \mathrm{C}$ y $-3,95{ }^{\circ} \mathrm{C}$ para Lomín (estepa), respectivamente.

En promedio, la temperatura media diaria del agua fue más fría y con máximas más bajas en el río Malleco, con presencia ribereña de $N$. dombeyi, que en el río Lomín, que drena áreas dominadas por estepa (Tabla II). Durante los meses de estudio no se registraron valores inferiores a $1^{\circ} \mathrm{C}$, sin embargo, se pudo observar una mayor ocurrencia de

TABLA I. Caracterización física de las subcuencas de estudio.

TABLE I. Physical characterization of the study catchment.

\begin{tabular}{|c|c|c|}
\hline & Lomín & Malleco \\
\hline \multirow[t]{2}{*}{ Localización } & $38^{\circ} 00^{\prime} 57,24^{\prime \prime} \mathrm{S}$ & $38^{\circ} 12^{\prime} 50,17^{\prime \prime} \mathrm{S}$ \\
\hline & $71^{\circ} 17^{\prime} 24,02 ” \mathrm{~W}$ & $71^{\circ} 48^{\prime} 21,68^{\prime \prime} \mathrm{W}$ \\
\hline Altitud (m) & 936 & 900 \\
\hline Área de drenaje $\left(\mathrm{km}^{2}\right)$ & 214,52 & 27,04 \\
\hline Altitud media de la subcuenca (ms.n.m) & 1.751 & 1.297 \\
\hline Altitud mínima de la subcuenca (ms.n.m) & 933 & 897 \\
\hline Altitud máxima de la subcuenca (ms.n.m) & 2.988 & 1.777 \\
\hline Pendiente media de la subcuenca & 0,32 & 0,24 \\
\hline Orden & 2 & 2 \\
\hline Ancho máximo (m) & 12 & 11,9 \\
\hline Profundidad media (m) & 0,6 & 0,8 \\
\hline Orientación & $\mathrm{E}$ & $\mathrm{E}$ \\
\hline Geología dominante & $\begin{array}{r}\text { Roca ígnea con } \\
\text { intrusiones sedimentarias }\end{array}$ & $\begin{array}{r}\text { Roca sedimentaria e } \\
\text { ígnea con intrusiones } \\
\text { sedimentarias }\end{array}$ \\
\hline
\end{tabular}

TABLA II. Estadística descriptiva de la temperatura media del aire y del agua $\left({ }^{\circ} \mathrm{C}\right)$ para los ríos Lomín y Malleco durante el periodo de estudio. (D.E. = desviación estándar).

TABLE II. Descriptive statistics of the air and water mean temperature $\left({ }^{\circ} \mathrm{C}\right)$ for Lomín and Malleco streams during studied period. $($ D.E. $=$ standard deviation).

\begin{tabular}{|c|c|c|c|c|}
\hline & \multicolumn{2}{|c|}{ Temperatura DEL AIRE } & \multicolumn{2}{|c|}{ Temperatura del Agua } \\
\hline & Lomín & Malleco & Lomín & Malleco \\
\hline Media & 8,73 & 8,15 & 8,80 & 8,14 \\
\hline D.E. & 5,96 & 5,54 & 2,80 & 2,65 \\
\hline Rango & 25,72 & 25,46 & 10,33 & 9,61 \\
\hline Máxima & 22,33 & 21,52 & 14,04 & 13,78 \\
\hline Mínima & $-3,95$ & $-3,39$ & 3,72 & 4,18 \\
\hline
\end{tabular}


temperaturas $<4^{\circ} \mathrm{C}(186,25 \mathrm{~h}$; equivalentes al 4,46\% del total de datos $) \mathrm{y} \geq 15^{\circ} \mathrm{C}(116,0 \mathrm{~h} ; 2,78 \%)$ en el río Lomín que en el Malleco (56,5 h (1,35\%) y 29,25 h (0,70\%)).

La variabilidad y diferencia diaria de los patrones térmicos del agua de ambos sitios se muestran en las Figuras 2 y 3 , respectivamente. Durante el periodo estival se observó que la temperatura media diaria fue en promedio $0,72{ }^{\circ} \mathrm{C}$ más baja en el río Malleco, detectándose la mayor diferencia en marzo con un valor de $2,2{ }^{\circ} \mathrm{C}$ más bajo. Por el contrario, hacia finales de otoño y comienzo del invierno se observaron temperaturas más cálidas, pero las diferencias fueron menores a $1{ }^{\circ} \mathrm{C}$ (Figs. 2a y 3a). La temperatura máxima diaria también fue más baja en el río Malleco promediando una disminución de $1,51{ }^{\circ} \mathrm{C}$, observándose la mayor diferencia entre marzo y abril con valores entre 0,49 y 3,81 ${ }^{\circ} \mathrm{C}$ más bajos. Sin embargo, hacia finales de otoño sólo se registraron tres días con temperaturas más cálidas que no superaron los $0,31^{\circ} \mathrm{C}$ (Figs. 2b y 3b). En gran parte del verano, la temperatura mínima diaria fue más alta en el río con bosque y las mayores diferencias se registraron durante febrero alcanzando valores de hasta $2,34^{\circ} \mathrm{C}$. Similar tendencia fue registrada hacia finales junio pero no superó los $1,57{ }^{\circ} \mathrm{C}$. Contrariamente, el río Lomín, con presencia de estepa, presentó mayores temperaturas mínimas desde mediados de abril hasta principio de junio, pero las diferencias fueron generalmente menores a $0,7{ }^{\circ} \mathrm{C}$ (Figs. $2 \mathrm{c}$ y 3c). Finalmente, la variabilidad y diferencia registradas entre los valores de temperatura máximos y mínimos diarios de ambos sitios se muestran en las Figuras $2 \mathrm{~d}$ y $3 \mathrm{~d}$, respectivamente. La tendencia a valores máximos más bajos y mínimos más altos de temperatura reducen el rango medio diario del río Malleco, con presencia boscosa de $N$. dombeyi en su ribera, en comparación con el río Lomín, con estepa. Este parámetro no superó $1^{\circ} \mathrm{C}$ en el primer caso, pero en el segundo alcanzó valores superiores a $4{ }^{\circ} \mathrm{C}$. Además, fue posible observar que el rango medio diario entre sitios mostró una clara estacionalidad con contrastes mayores en verano y pequeñas diferencias a mediados del otoño. Diferencias significativas entre sitios $(\mathrm{n}=192$ días; $P<0,05)$ se registraron para los parámetros térmicos del agua: media, máxima y rango medio diario.

Esta diferencia registrada en la variabilidad espaciotemporal de la temperatura media diaria también se manifiesta en los patrones de grados-día mensual y
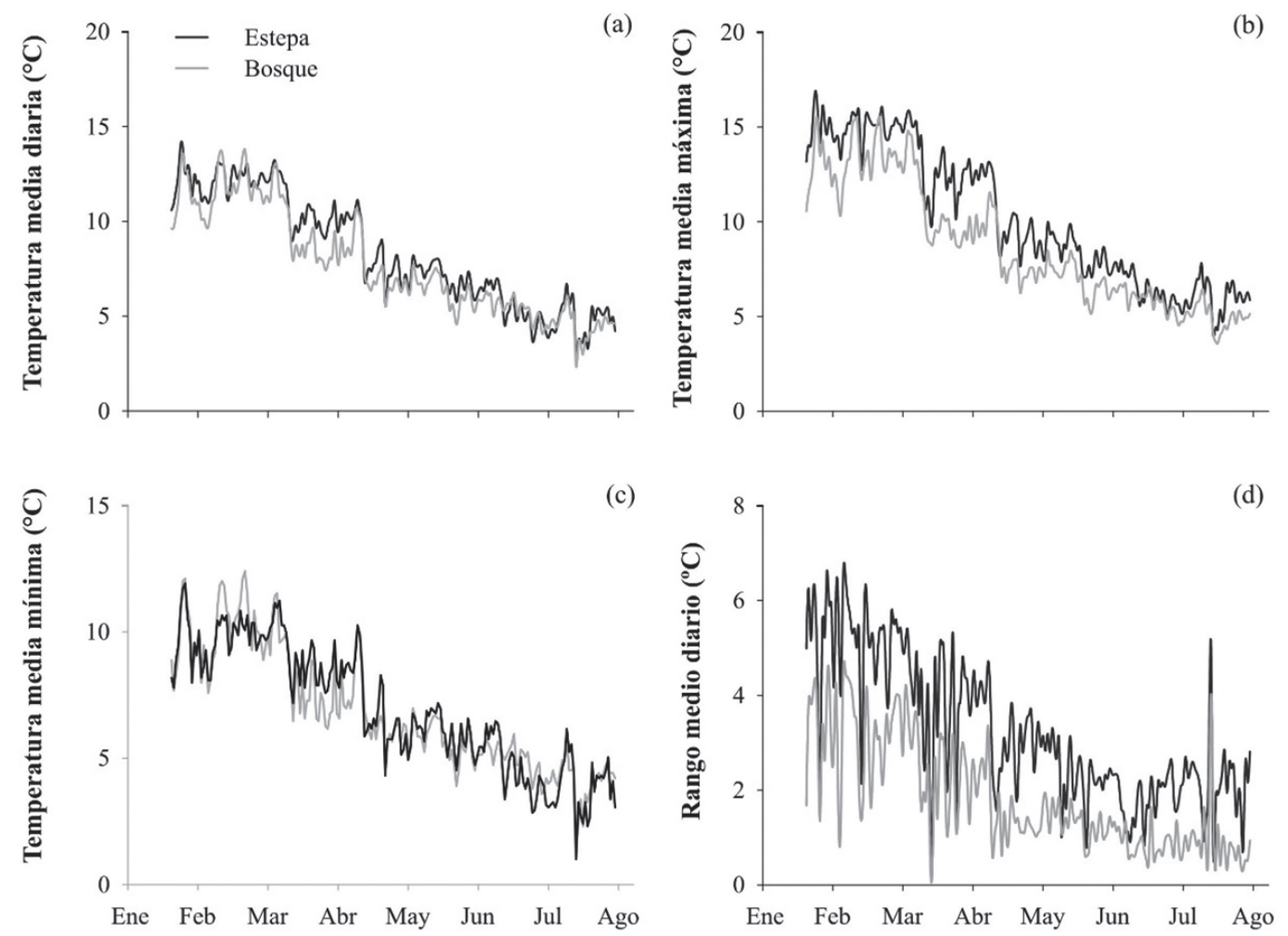

FiguRA 2. Variabilidad térmica diaria del agua para la: (a) temperatura media, (b) temperatura media máxima, (c) temperatura media mínima y (d) rango medio de temperaturas de los ríos Lomín, con estepa y Malleco, con bosque nativo.

FIGURE 2. Daily temperature variability of water for the: (a) mean temperature, (b) mean maximum temperature, (c) mean minimum temperature and (d) mean range of the rivers Lomín with steppe and Malleco with native forest. 


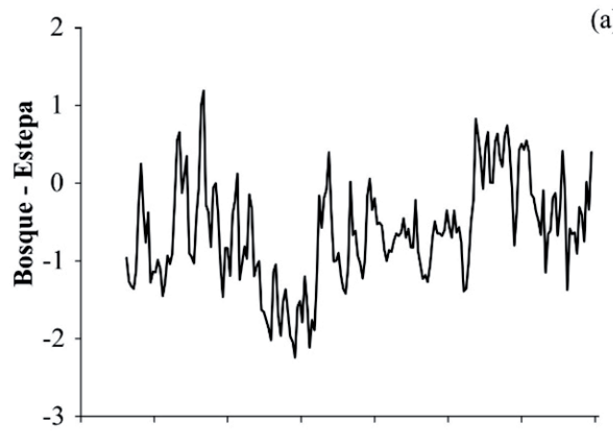

(a)
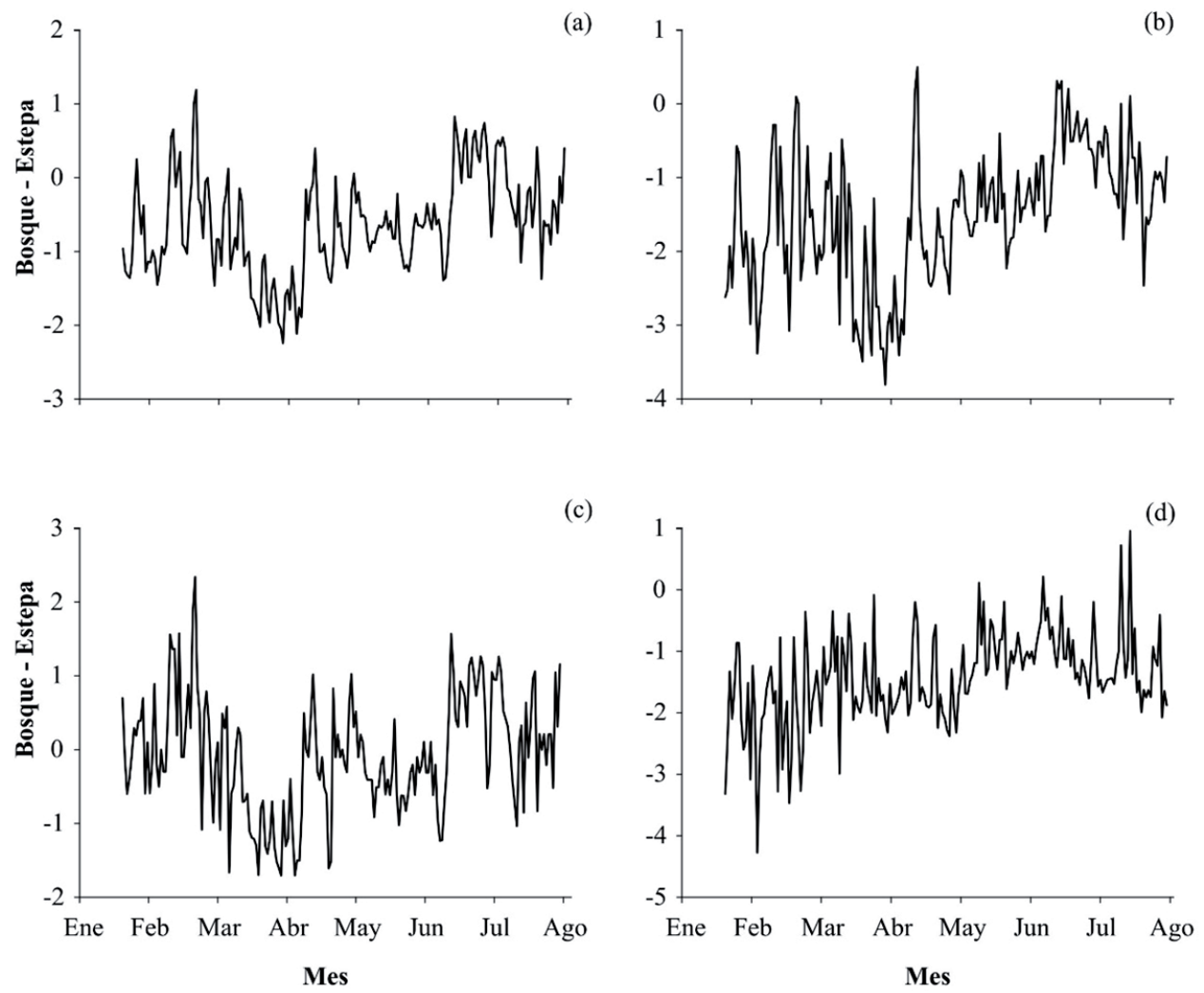

Figura 3. Diferencia entre la temperatura del agua registrada en el río Malleco (bosque nativo) y el río Lomín (estepa): (a) temperatura media, (b) temperatura media máxima, (c) temperatura media mínima y (d) rango medio diario.

FIGURE 3. Difference between the water temperatures recorded in the river Malleco (native forest) and river Lomín (steppe) for: (a) mean temperature, (b) average maximum temperature, (c) average minimum temperature and (d) mean daily range.
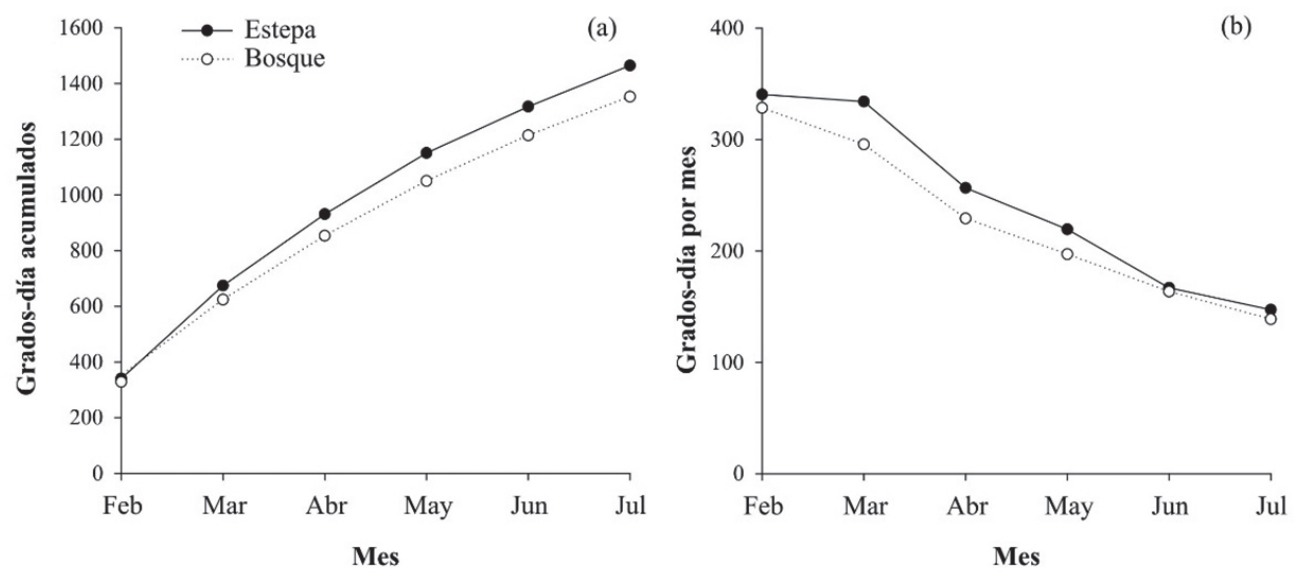

FiguRA 4. Grados-día acumulados (a) y grados-día por mes (b), para los ríos Lomín, con estepa, y Malleco, con bosque nativo.

FigURE 4. Cumulative degree-days (a) and monthly average degree-days (b), for rivers Lomín, with steppe and Malleco with native forest. 
acumulado. La variabilidad de los grados-día acumulados alcanzó valores de 1.464 y 1.352 , para los ríos Lomín y Malleco, respectivamente (Fig. 4a). Los grados-día mensual son función de las temperaturas estivales. No obstante, en junio se pudo observar que los valores tienden a igualarse debido a que la temperatura media diaria es mayor en el río Malleco, con presencia ribereña de N. dombeyi (Fig. 4b).

La relación entre la temperatura del agua y del aire en ambos casos presentó una correlación altamente significativa $(P<0,001)$, no obstante el coeficiente de correlación fue levemente más bajo en el sitio con estepa $(\mathrm{R}=0,80$ Lomín, $\mathrm{R}=0,84$ Malleco). A escala temporal diaria, el ciclo de temperatura del aire mostró ser más fluctuante en el río Lomín, con diferencias significativas sólo para él día más frío $(\mathrm{U}=3.641 ; P<0,01)$ (Fig. 5). Además, existió un leve retraso en la temperatura mínima y adelanto en la máxima en el río con bosque. Sin embargo, en relación al ciclo de temperatura del agua, se encontraron diferencias significativas entre los sitios en ambas fechas. Para el día más cálido $(\mathrm{U}=3.093 ; P<0,001)$, la temperatura máxima se adelantó aproximadamente $1 \mathrm{~h}$ en el río con bosque nativo $(16: 30 \mathrm{~h})$ y permaneció constante durante aproximadamente $2 \mathrm{~h}$ (Fig. 5a). Contrariamente, para el día más frío (U= 2.104,5; $P<0,001)$, la temperatura mínima tuvo un retraso de $1 \mathrm{~h} 15 \mathrm{~min}$ en el río con bosque nativo y permaneció constante durante aproximadamente $3 \mathrm{~h}$ (Fig. 5b).
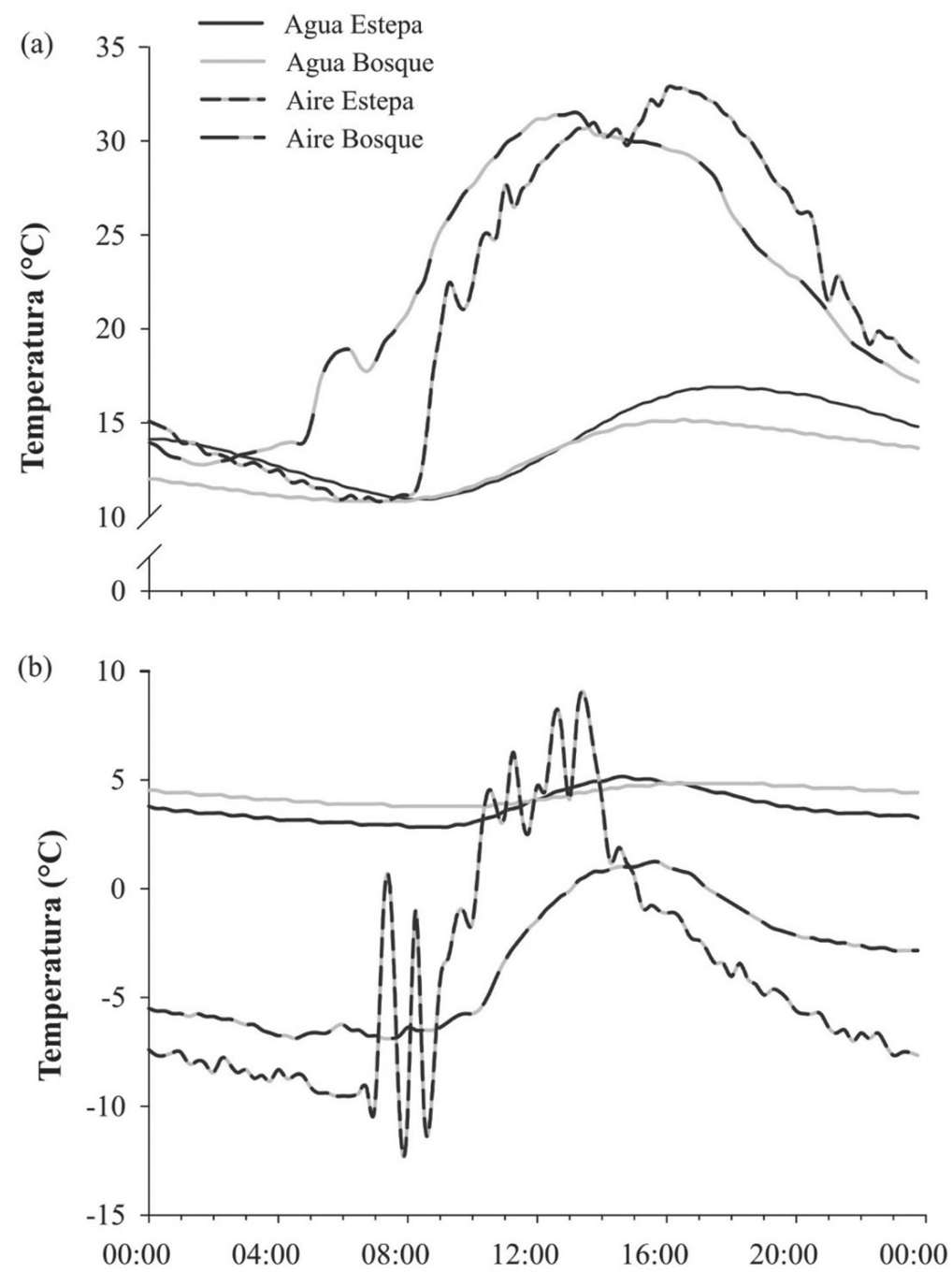

Hora

Figura 5. Ciclo diario de la temperatura del aire y del agua para: (a) día más cálido y (b) día más frío registrado en el período de estudio. FIGURE 5. Diurnal cycle of air and water temperature for: (a) warmest day and (b) coldest day in the study period. 


\section{DISCUSIÓN}

Los resultados de este estudio revelaron diferencias significativas en la temperatura media, máxima y rango medio diario entre el sitio con presencia de $N$. dombeyi y el sitio con estepa en su ribera, lo que indica que la vegetación ribereña nativa tiene un rol importante en controlar la temperatura de los ríos andinos de la zona centro-sur de Chile. Asimismo, la utilización de datos con alta resolución (15 min) permitió reconocer detalladamente el ciclo diario de la temperatura del agua durante los extremos de temperatura del aire (mínima y máxima). Estos resultados son concordantes con investigaciones previas desarrolladas principalmente en Europa y Norteamérica (Johnson 2004, Malcolm et al. 2004, Webb \& Crisp 2006, Malcolm et al. 2008, Brown et al. 2010).

Cuando se trabaja con una temporalidad de duración limitada, los factores a escala de tramo de río parecen ser importantes modeladores de la temperatura (Malcolm et al. 2004, Pedreros et al. 2013). Así, la presencia y/o ausencia de vegetación ribereña perenne mostró ser crítica para el régimen térmico de los ríos andinos Lomín y Malleco. La variabilidad térmica del tramo con presencia de $N$. dombeyi fue $\sim 0,6{ }^{\circ} \mathrm{C}$ más fría que el tramo con presencia de estepa, este valor fue levemente más alto que los reportados para ríos de montaña en Escocia y Norte de Gales (Crisp 1997, Webb \& Crisp 2006). Estas desigualdades entre localidades pueden ser atribuidas a características geomorfológicas, así como a la combinación de condiciones climáticas durante la realización de los estudios (Brown \& Hannah 2008). De acuerdo a Brown (1969) las características ribereñas como densidad y tipo vegetación varía entre sitios, lo cual puede contribuir a las diferencias térmicas (Moore et al. 2005b).

La presencia de vegetación ribereña perenne de $N$. dombeyi redujo $\sim 4{ }^{\circ} \mathrm{C}$ la temperatura máxima diaria, lo cual se asemeja a los resultados obtenidos por Hannah et al. (2008). Sin embargo, otros estudios describen que esta reducción puede ser aún mayor (Caissie 2006, Webb \& Crisp 2006, Brown et al. 2010), pero depende directamente de la conformación del dosel y su orientación al cauce (Hannah et al. 2008). Esta diferencia de temperatura entre ríos con vegetación ribereña contrastante se asocia a la sombra generada por el dosel, la cual disminuye la entrada directa de radiación solar neta (Johnson \& Jones 2000, Malcolm et al. 2004, Moore et al. 2005b, Hannah et al. 2008, Brown et al. 2010), fuente principal de energía calórica en ríos de bajo orden (Isaak \& Hubert 2001).

Si bien los ríos con presencia ribereña boscosa son más fríos que aquellos con presencia de estepa durante los meses de verano - por efecto de la sombra- algunos estudios han reportado que en invierno la temperatura es más alta en tramos con presencia de bosque, debido a que el dosel actúa como aislante, manteniendo la temperatura del aire y reduciendo la perdida de radiación de onda larga (Webb
\& Zhang 2004, Webb \& Crisp 2006, Hannah et al. 2008, Brown et al. 2010). Esta afirmación concuerda parcialmente con los resultados encontrados en esta investigación, ya que a fines del otoño y comienzo del invierno se observaron temperaturas significativamente más altas en el río con presencia de bosque, sin embargo, se requieren de nuevas aproximaciones para capturar la variabilidad anual que permita realizar una caracterización a nivel estacional.

La leve diferencia en la relación de temperatura aireagua encontrada en este estudio fue similar a la reportada por Brown et al. (2010) para pequeños ríos en Inglaterra. Estos autores lo atribuyen a que, en ríos con presencia de páramo, la dinámica térmica está altamente ligada a la variabilidad diaria y anual de la condición meteorológica, por lo que la radiación solar incidente, sumado a los flujos de calor sensible y latente, conforman los mayores componentes del balance térmico en dichos sistemas (Hannah et al. 2008). Adicionalmente, la baja capacidad térmica que presenta el río Lomín (Pedreros et al. 2013) beneficia la ocurrencia de fluctuaciones térmicas más altas. Finalmente, el análisis del ciclo diario de temperatura del agua mostró claras diferencias entre sitios, observándose que la presencia del dosel de $N$. dombeyi redujo la entrada de la radiación solar directa al cauce en época de verano, mientras que en otoño e invierno la reducción de la radiación de onda larga bajo el dosel favoreció la ocurrencia de temperaturas más altas. Por lo tanto, la disminución de radiación solar neta en los meses de verano, así como la capacidad de generar condiciones microclimáticas durante el invierno, le confieren a la vegetación ribereña una función relevante en el control de la dinámica térmica en los ríos (Moore et al. 2005a, Hannah et al. 2008).

La presente investigación permitió demostrar la importancia que tiene la sombra generada por $N$. dombeyi en el control de la temperatura de ríos andinos del centro-sur de Chile. Su presencia redujo la temperatura diaria del agua durante la época estival, mientras que hacia finales de otoño y comienzos de invierno generó una condición aislante que mantuvo la temperatura del cauce. Asimismo, la baja presión antrópica de los ríos estudiados posibilitó que los patrones térmicos encontrados respondan a la variabilidad natural individual de cada sitio. Igualmente, la utilización de intervalos de tiempo de alta resolución (15 min) permitió explorar detalladamente el efecto que tiene la presencia de $N$. dombeyi sobre el ciclo diario y los extremos (día más cálido y día más frío) de temperatura del agua.

Aunque no es nueva la idea de mostrar la importancia de la vegetación ribereña sobre el régimen térmico de los ríos, la investigación toma relevancia considerando que la región posee grandes valores ecológicos y ambientales que actualmente se encuentran amenazados por la continua fragmentación del bosque nativo, sin tener en cuenta el importante rol que juega en el balance térmico de los ríos que conservan una alta biodiversidad. Por lo que la 
evaluación de nuevos sitios; particularmente ríos con escasa perturbación; y una serie temporal más extensa, no sólo contribuirán a avanzar en la comprensión del efecto de la vegetación ribereña nativa sobre la variabilidad espaciotemporal del régimen térmico en ríos andinos, sino también en la importancia de esta variable en la ecología fluvial. Además, la conservación y restauración de la vegetación nativa aledaña a los ríos podría ser relevante para mitigar los nuevos regímenes de temperatura proyectados según el calentamiento global.

\section{AGRADECIMIENTOS}

El presente trabajo fue financiado por los Proyectos: Fondecyt de Iniciación 11100119 y CRHIAM/CONICYT/ FONDAP 15130015. También se desea agradecer al Sr. César Pedreros M. por su colaboración en terreno.

\section{REFERENCIAS}

Acuña, V., A. Wolf, U. Uehlinger \& K. Tockner. 2008. Temperature dependence of stream benthic respiration in an Alpine river network under global warming. Freshwater Biology 53: 2076-2088.

Aguayo, M., A. Pauchard, G. Azócar \& O. Parra. 2009. Land use change in the south central Chile at the end of the 20(th) century. Understanding the spatio-temporal dynamics of the landscape. Revista Chilena de Historia Natural 82: 361-374.

Allan, J. D. \& M. M. Castillo. 2007. Stream Ecology Structure and function of running waters. Springer, Dordrecht, Netherlands. $436 \mathrm{pp}$.

Arscott, D.B., K. Tockner \& J.V. Ward. 2001. Thermal heterogeneity along a braided floodplain river (Tagliamento River, northeastern Italy). Canadian Journal of Fisheries and Aquatic Sciences 58: 23592373.

Berner, E.K. \& R.A. Berner. 1996. Global environment: Water, air and geochemical cycles. Prentice Hall, New Jersey. $376 \mathrm{pp}$.

Brown, G.W. 1969. Predicting Temperatures of Small Streams. Water Resources Research 5: 68-75.

Brown, L.E., L. CoOper, J. Holden \& S.J. Ramchunder. 2010. A comparison of stream water temperature regimes from open and afforested moorland, Yorkshire Dales, northern England. Hydrological Processes 24: 3206-3218.

BRown, L.E. \& D.M. HANNAH. 2008. Spatial heterogeneity of water temperature across an alpine river basin. Hydrological Processes 22: 954-967.

CAissie, D. 2006. The thermal regime of rivers: a review. Freshwater Biology 51: 1389-1406.

Cisternas, M., P. Martínez, C. Oyarzún \& P. Debels. 1999. Caracterización del proceso de reemplazo de vegetación nativa por plantaciones forestales en una cuenca lacustre de la Cordillera de Nahuelbuta, VIII Región, Chile. Revista Chilena de Historia Natural 72: 661-676.
CRISP, D.T. 1997. Water temperature of Plynlimon streams. Hydrology and Earth System Sciences 1: 535-540.

Danehy, R.J., C.G. Colson, K.B. Parrett \& S.D. Duke. 2005. Patterns and sources of thermal heterogeneity in small mountain streams within a forested setting. Forest Ecology and Management 208: 287-302.

Fox, J. 2005. The R Commander: A Basic Statistics Graphical User Interface to R. Journal of Statistical Software 14: 1-42.

Gasith, A. \& V.H. Resh. 1999. Streams in Mediterranean Climate Regions: Abiotic Influences and Biotic Responses to Predictable Seasonal Events. Annual Review of Ecology and Systematics 30: 51-81.

Hannah, D.M., I.A. Malcolm, C. Soulsby \& A.F. Youngson. 2008. A comparison of forest and moorland stream microclimate, heat exchanges and thermal dynamics. Hydrological Processes 22: 919-940.

IsAAK, D.J. \& W.A. HuBERT. 2001. A hypothesis about factors that affect maximum summer stream temperatures across montane landscapes. Journal of the American Water Resources Association 37: 351-366.

Johnson, S.L. 2003. Stream temperature: scaling of observations and issues for modelling. Hydrological Processes 17: 497-499.

JohNSON, S.L. 2004. Factors influencing stream temperatures in small streams: substrate effects and a shading experiment. Canadian Journal of Fisheries and Aquatic Sciences 61: 913-923.

Johnson, S.L \& J.I. Jones. 2000. Stream temperature responses to forest harvest and debris flows in western Cascades Oregon. Canadian Journal of Fisheries and Aquatic Sciences 57: 30-39.

Link, O., A. Huerta, A. Stehr, A. Monsalve, C. Meier \& M. Aguayo. 2012. The solar-to-stream power ratio: A dimensionless number explaining diel fluctuations of temperature in mesoscale rivers. River Research and Applications 29: 792-803.

Link, O., A. Monsalve, A. Stehr, A. García \& R. Urrutia. 2009. Régimen térmico del río Itata. En: O. Parra, J.C. Castilla, H. Romero, R. Quiñones, A Camaño (eds.), La cuenca hidrográfica del río Itata, aportes científicos para su gestión sustentable, pp. 44-57. Editorial Universidad de Concepción, Concepción, Chile.

MacDonald, J.S., E.A. MacIsaac \& H.E. Herunter. 2003. The effect of variable-retention riparian buffer zones on water temperatures in small headwater streams in subboreal forest ecosystems of British Columbia. Canadian Journal of Forest Research 33: 1371-1382.

Malcolm, I.A., D.M. Hannah, M.J. Donaghy, C. Soulsby \& A.F. YounGSON. 2004. The influence of riparian woodland on the spatial and temporal variability of stream water temperatures in an upland salmon stream. Hydrology and Earth System Sciences 8: 449-459.

Malcolm, I.A., C. Soulsby, D.M. Hannah, P.J. Bacon, A.F. Youngson \& D. Tetzlaff. 2008. The influence of riparian woodland on stream temperatures: implications for the performance of juvenile salmonids. Hydrological Processes 22: 968-979.

Mardones, M., E. Jaque \& D. Zaio. 1992. Geomorfología de la hoya hidrográfica del río Bio-Bío. Instituto Geográfico Mlitar, Santiago, Chile. 631 pp. 
Monsalve, A., O. LinK, A STehr. 2012. Régimen térmico de ríos: Desarrollo, verificación y aplicación de un modelo numérico. Tecnología y Ciencias del Agua 3: 41-56.

Moore, R.D., D.L. Spittlehouse \& A. Story. 2005a. Riparian microclimate and stream temperature response to forest harvesting: A review. Journal of the American Water Resources Association 41: 813-834.

Moore, R.D., P. Sutherland, T. Gomi \& A. Dhakal. 2005 b. Thermal regime of a headwater stream within a clearcut, coastal British Columbia, Canada. Hydrological Processes 19: 2591-2608.

Olden, J.D. \& R.J. NAIMAN. 2010. Incorporating thermal regimes into environmental flows assessments: modifying dam operations to restore freshwater ecosystem integrity. Freshwater Biology 55: 86-107.

Pedreros, P., M. Guevara, R. Figueroa, A. Araneda, A. Stehr, O. LinK \& R. Urrutia. 2013. Comportamiento térmico en ríos mediterráneos alto-andinos de la zona centro-sur de Chile. Limnetica 32: 87-96.

Poole, G.C. \& C.H. Berman. 2001. An ecological perspective on in-stream temperature: Natural heat dynamics and mechanisms of human-caused thermal degradation. Environmental Management 27: 787-802.

QuinN, J. \& A. Wrightstow. 2008. Stream size influences stream temperature impacts and recovery rates after clearfell logging. Forest Ecology and Management 256: 21012109 .
Rutherford, J.C., N.A. Marsh, P.M. Davies \& S.E. Bunn. 2004. Effects of patchy shade on stream water temperature: how quickly do small streams heat and cool? Marine and Freshwater Research 55: 737-748.

Sinokrot, B.A. \& H.G. Stefan. 1993. Stream temperature dynamics: Measurements and modeling. Water Resources Research 29: 2299-2312.

Torrejón, F. \& M. Cisternas. 2002. Alteraciones del paisaje ecológico araucano por la asimilación mapuche de la agroganadería hispano-mediterránea (siglos XVI y XVII). Revista Chilena de Historia Natural 75: 729-736.

Vannote, R.L. \& B.W. SweEney. 1980. Geographic Analysis of Thermal Equilibria: A Conceptual Model for Evaluating the Effect of Natural and Modified Thermal Regimes on Aquatic Insect Communities. The American Naturalist 115: 667-695.

Webi, B.W. \& D.T. Crisp. 2006. Afforestation and stream temperature in a temperate maritime environment. Hydrological Processes 20: 51-66.

Webb, B.W., D.M. Hannah, R.D. Moore, L.E. Brown \& F. Nobilis. 2008. Recent advances in stream and river temperature research. Hydrological Processes 22: 902-918.

WebB, B.W. \& Y. Zhang. 1997. Spatial and seasonal variability in the components of the river heat budget. Hydrological Processes 11: 79-101.

WebB, B.W. \& Y. Zhang. 2004. Intra-annual variability in the non-advective heat energy budget of Devon streams and rivers. Hydrological Processes 18: 2117-2146.

Recibido: 05.10.15

Aceptado: 05.05.16 